\title{
Comparison of total hip and knee joint replacement in patients with rheumatoid arthritis and osteoarthritis: a nationwide, population-based study
}

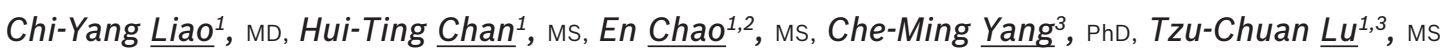

INTRODUCTION Patients with rheumatoid arthritis (RA) and osteoarthritis (OA) may require total hip replacement (THR) or total knee replacement (TKR). The present study aimed to compare the demographic characteristics and medical costs of RA and OA patients from Taiwan who underwent either THR or TKR.

METHODS The medical records of patients who had undergone THR or TKR from 1 January 1996 to 31 December 2010 were obtained from the Taiwan National Health Insurance Research Database (NHIRD). In all, we found 49 and 146 RA patients who received THR and TKR, respectively, and 1,191 and 6,574 OA patients who received THR and TKR, respectively. The gender, age, Charlson comorbidity index $(\mathrm{CCl})$, hospital grade, age at registration in the catastrophic illness dataset, and medical utilisation costs of the different groups were compared.

RESULTS There were statistically significant differences in age, $\mathrm{CCl}$ score, drug costs and surgery costs between RA and OA patients. Joint replacement incidence was lower in RA patients than in OA patients, and among patients who underwent THR, total medical costs incurred were higher for RA patients than OA patients. RA patients who underwent THR incurred a significantly greater total medical utilisation cost in the outpatient department ( 3 months before surgery and 12 months after surgery) than OA patients who underwent THR.

CONCLUSION Analysis of Taiwan NHIRD with regard to patients who had undergone either THR or TKR indicated that RA patients were younger than OA patients, and that significantly more medical resources were used for RA patients before, during and after hospitalisation for these procedures.

Keywords: National Health Insurance Research Database, osteoarthritis, rheumatoid arthritis, total hip replacement, total knee replacement

\section{INTRODUCTION}

Rheumatoid arthritis (RA) is a chronic autoimmune disease that usually leads to persistent and painful disability in multiple joints, progressive deterioration of joint structures, and the use of a significant amount of medical resources. ${ }^{(1,2)}$ Current research estimates that RA affects about $1 \%$ of people worldwide. ${ }^{(3)}$ Native Americans have a high prevalence of RA (5\%-6\%), while Caribbean and Asian populations have a low prevalence of RA. ${ }^{(4,5)}$ RA affects more than 2,000,000 adults in the United States $(\sim 1 \%), 491,000$ in Australia ( $2 \%-3 \%)$, 300,000 in Canada ( 1\%), and 110,000 in Taiwan $(\sim 0.5 \%) .{ }^{(6)-8)}$ In Australia, about $1.4 \%$ and $2.6 \%$ of RA patients have undergone total hip replacement (THR) and total knee replacement (TKR), respectively, from 1999 to 2003. ${ }^{(9)}$ In Canada, between 2004 and 2005, RA patients accounted for less than $4 \%$ of all THRs performed and $1 \%$ of primary THRs. ${ }^{(10)}$ In 2005 , RA patients accounted for $4 \%$ of all TKRs performed and $2 \%$ of primary TKRs in the United Kingdom. ${ }^{(11)}$

The present study is a longitudinal, population-based study of 1,000,000 RA and osteoarthritis (OA) patients in Taiwan who received either THR or TKR from 1 January 1996 to 31 December 2010. Han Chinese comprises over $92 \%$ of the population in Taiwan and mainland China, over $74 \%$ of the population in Singapore, and $24.5 \%$ of the population in Malaysia. ${ }^{(12)}$ To the best of our knowledge, this is the first study comparing THR and TKR in such a large number of RA and OA patients over a period of 15 years. The purpose of the present study was to compare the utilisation of inpatient and outpatient medical resources in RA and OA patients who received either THR or TKR. The data obtained can be used by the government and private insurance companies to collect insurance premiums, and is especially useful if the government wishes to set up their own health insurance system.

\section{METHODS}

The Taiwan National Health Insurance Research Database (NHIRD) covers all inpatient medical benefit claims and includes data on approximately $98 \%$ of Taiwan's population ( 23 million people). The claims data is administered by the Bureau of National Health Insurance (BNHI), the sole purchaser in Taiwan. In other words, this dataset is the most comprehensive and reliable data source available for the present study.

For random sampling, we used the NHIRD Longitudinal Health Insurance Database (LHID) 2005. LHID 2005 is a subset database that contains the data of all the original claims made for 1 million beneficiaries, who were randomly sampled from the 2005 Registry for Beneficiaries (ID) of the NHIRD and enrolled from 1 January 2005 to 1 January 2006. There was no significant difference in gender distribution in LHID 2005 and

\footnotetext{
'Department of Orthopaedics, Sung Shan Branch, Tri-Service General Hospital, National Defense Medical Center, ${ }^{2}$ Department of Health Promotion and Health Education, Taiwan Normal University, ${ }^{3}$ School of Health Care Administration, Taipei Medical University, Taiwan

Correspondence: Dr Tzu-Chuan Lu, Consultant, Department of Orthopaedics, Sung Shan Branch, Tri-Service General Hospital, No 131 Chien-Kang Road, Taipei, Taiwan. lutz.chung@msa.hinet.net
} 
the original NHIRD $\left(\chi^{2}=0.008, p=0.05,2\right.$ degrees of freedom $)$. LHID includes the registries of contracted medical facilities and board-certified physicians, and details of in- and outpatient medical utilisation for all patients in Taiwan aged $>15$ years. It also provides the operation procedure and diagnosis codes for each patient, based on the International Classification of Disease, Ninth Revision, Clinical Modification (ICD-9-CM) ${ }^{(13)}$ As the present study used anonymised secondary data, the ethics committee of our institution waived the requirement for informed consent.

Our study sample consisted of all patients who were identified to have undergone primary THR (ICD-9-CM procedure code 81.51 ) or primary TKR (ICD-9-CM procedure code 81.54 ) in LHID. These two procedure codes were among the five other surgical procedure codes from 1996 to 2010 . There were a total of 3,021 THRs and 7,490 TKRs. We excluded patients whose records indicated any of the following: misinterpreted data, unreasonably low total medical costs, IDs presenting more than twice, history of haemophilia, history of joint infection, history of malignant bone tumour with joint reconstruction procedure or procedures concomitant with another major operation, and revision THRs or TKRs. This led to a total of 2,843 THR patients and 7,352 TKR patients.

From the 2,843 THR patients and 7,352 TKR patients, patients with RA were identified using the ICD-9-CM diagnosis code 714.0, in conjunction with the procedure code for THR or TKR. Using this method, we identified 66 and 178 RA patients who underwent THR and TKR, respectively. These identified RA patients were then checked against the NHIRD-HV dataset (i.e. the catastrophic illness dataset) to confirm the diagnosis of RA. After this check, the patient count was reduced to 52 and 153 RA patients who underwent THR and TKR, respectively. In the final step, we excluded patients with ICD-9-CM procedure codes 81.51 and/or 81.54, if the codes were presented twice during one admission, which represents a simultaneous bilateral arthroplasty. In all, there were 49 RA patients who had a single THR and 146 RA patients who had a single TKR during one admission (Fig. 1).

For the comparison group, the records of OA patients who had knee and hip procedures from the database were extracted in a manner similar to that used for the RA patients - from the 2,843 primary THR and 7,352 TKR procedures identified, we used the same exclusion criteria as that used in the identification of RA patients. Patients with OA were identified using the ICD-9-CM diagnosis codes 715.00-715.98, together with the procedure codes for THR or TKR. Again, we excluded patients who had the ICD-9-CM procedure codes 81.51 or 81.54 presented twice during one admission. Ultimately, there were 1,191 OA patients who had a single THR and 6,574 OA patients who had a single TKR during one admission.

We compared two dimensions of medical resource utilisation in both RA and OA patients who underwent THR or TKR: (a) demographic data and medical resource utilisation during hospitalisation; and (b) total outpatient medical resource utilisation 3 months before surgery, and 3, 4-6 and 7-12 months after surgery. We stratified our study sample into three consecutive

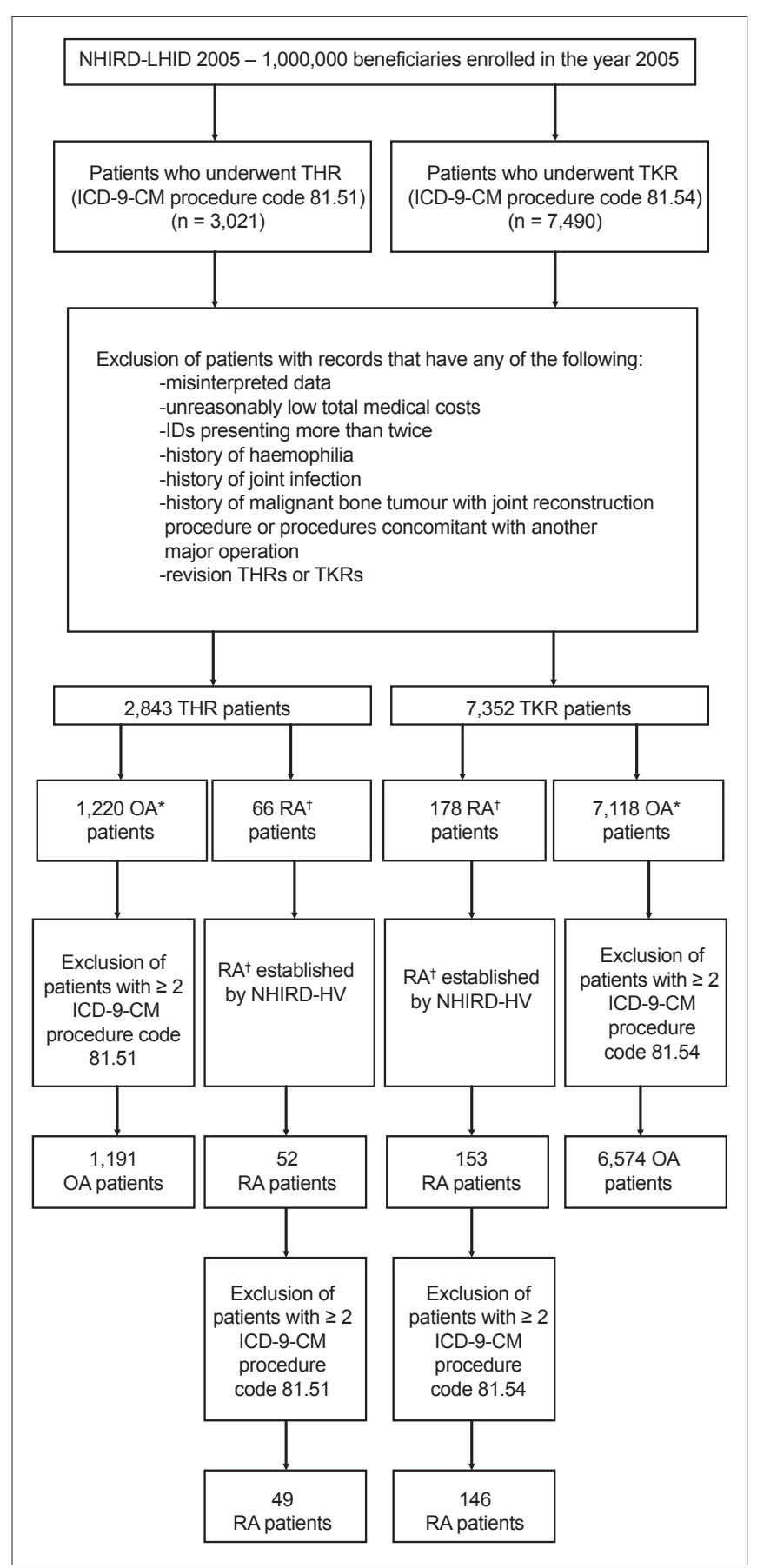

Fig. 1 Flowchart shows the identification process of rheumatoid arthritis (RA) and osteoarthritis (OA) patients for the present study from a pool of $1,000,000$ patients (data extracted from 1 January 1996 to 31 December 2010) who underwent either total hip replacement (THR) or total knee replacement (TKR). *Identified by ICM-9-CM diagnosis codes $715.00-$ 715.98. IIdentified by ICM-9-CM diagnosis code 714.0. NHIRD: National Health Insurance Research Database; LHID: Longitudinal Health Insurance Database

5-year periods (i.e. 1996-2000, 2001-2005 and 2006-2010) for analysis. All dollar values at the end of each year were adjusted to the 2010 Taiwan currency values. Hospital charges were then converted from Taiwan dollars to United States dollars at an exchange rate of $31.64: 1$, based on the average exchange rate from 1996 to 2010.

Statistical analysis was conducted using the Statistical Package for the Social Sciences version 17.0 (SPSS Inc, 
Chicago, IL, USA). Descriptive and summary statistics were performed for demographic characteristics and outcomes. Independent $t$-test was used to analyse the medical utilisation of RA and OA patients who underwent THR or TKR. We used repeated measures analysis of variance (rANOVA) to analyse factors associated with the total cost of outpatient medical utilisation, according to time (i.e. 3 months before surgery, and 3, 4-6 and 7-12 months after surgery), patient group (i.e. patients with $\mathrm{OA}$ and those with RA), and patient interaction for each procedure (THR and TKR). A two-sided p-value $<0.05$ was considered statistically significant.

\section{RESULTS}

Based on the claims data (for in-hospital medical resource utilisation) of 1,000,000 patients in Taiwan over 15 years, a total of 49 THRs and 146 TKRs were performed for RA patients, and for OA patients, 1,191 THRs and 6,574 TKRs. Table I shows the gender, age, Charlson comorbidity index $(\mathrm{CCl}),{ }^{(14,15)}$ average length of stay (ALOS), hospital grade, cost of administered drugs, cost of laboratory examinations, cost of surgery and total medical costs for all patients, stratified according to the three aforementioned time periods (i.e. 1996-2000, 2001-2005 and 2006-2010).

Comparing among the three time periods, the gender ratios of the RA and OA patients who had undergone THR were not significantly different (all $p>0.05$ ). However, while the gender ratios between RA and OA patients who had undergone TKR were not significantly different in 2001-2005 and 2006-2010 (both $\mathrm{p}>0.05$ ), it was significantly different in 1996-2000 $(p=0.04)$. Also, with regard to THR in 1996-2000, there was a significant difference in mean age between RA and OA patients (44.8 years vs. 58.6 years; $p=0.01$ ). According to time period, we found significant differences between the mean age of the RA and OA patients who underwent TKR (54.4 years, 59.6 years and 64.0 years vs. 66.9 years, 68.6 years and 69.9 years, in the order 1996-2000, 2001-2005 and 2006-2010, respectively; all $\mathrm{p}<0.01)$. Regarding THR in all three time periods, the $\mathrm{CCl}$ scores of RA patients were significantly greater than those of OA patients (all $p<0.01$ ). The same was observed with regard to patients who underwent TKR, with the $\mathrm{CCl}$ scores of RA patients found to be significantly greater than those of OA patients in all three time periods (all $\mathrm{p}<0.01$ ).

The three hospital grades in Taiwan consist of medical centre (> 500 beds), regional hospital (251-500 beds) and district hospital (21-250 beds). In our analysis of hospital grades in all three time periods, we found no significant difference between the RA and OA patients who underwent THR (all $p>0.05$ ). There was also no significant difference between the RA and OA patients who underwent TKR in 1996-2000 and 2001-2005 (both $p$ > 0.05), but there was a marginally significant difference between the RA and OA patients who underwent TKR in 2006-2010 ( $p=0.04)$. In both the THR and TKR groups, there was no significant difference in the ALOS of RA and OA patients in all three time periods (all $p>0.05)$. There was a significant difference between the RA and OA patients who underwent THR with respect to the rate of readmission within 14 days in 2001-2005 ( $p=0.02$ ). There was also a significant difference between RA and OA patients who underwent TKR with respect to the rate of readmission at 31-90 days in 2001-2005 ( $<<0.01)$.

For the THR group, the mean cost of laboratory examinations was significantly greater for RA patients than OA patients in 1996-2000 (\$164 vs. \$88; $p=0.01$ ). However, in the TKR group, RA and OA patients had similar mean laboratory examination costs in all three time periods. The same trend was observed for the mean cost of administered drugs in the THR group - the mean cost was significantly greater for RA patients than OA patients in 1996-2000 (\$218 vs. \$122; $p=0.02$ ); however, RA and OA patients who underwent TKR had similar mean drug costs in all three time periods. In the THR group in 2001-2005, the mean cost of surgery was significantly greater for RA patients than OA patients ( $\$ 934$ vs. $\$ 875 ; p<0.01$ ). In the TKR group in 2006-2010, the mean cost of surgery was significantly lower for RA patients than OA patients ( $\$ 862$ vs. $\$ 909 ; p<0.01$ ). The mean total medical cost in the THR group was significantly greater for RA patients than for OA patients in 1996-2000 (\$4,435 vs. $\$ 3,542 ; p=0.01$ ) and 2001-2005 ( $\$ 3,906$ vs. $\$ 3,379 ; p=0.03$ ). In the TKR group in 2006-2010, the mean total medical cost was significantly lower for RA patients than for OA patients $(\$ 3,626$ vs. $\$ 3,772 ; p=0.01$ ).

Table II shows the results of the rANOVA of the total outpatient medical costs before and after surgery. In all three time periods, we found no significant within-subject effects in both the RA patients who underwent THR and the RA patients who underwent TKR (all $p>0.05$ ). However, in all three time periods, there were significant within-subject effects in both the OA patients who underwent THR and the OA patients who underwent TKR (all $\mathrm{p}<0.01$ ).

The results of the pairwise comparison tests of these groups of patients for 3 months before surgery, and 3, 4-6, and 7-12 months after surgery are also shown in Table II. In all three time periods, there was no significant difference between the RA patients who underwent THR and the RA patients who underwent TKR 3 months before surgery (all $p>0.05$ ). There were, however, significant differences with respect to medical costs between the OA patients who underwent THR at 4-6 months after surgery in all three time periods (all $p<0.01$ ), and at $7-12$ months after surgery in 2006-2010 ( $p<0.01)$. There were also significant differences with respect to medical costs between OA patients who underwent TKR at 3 months after surgery, 4-6 months after surgery and 7-12 months after surgery in all three time periods (all $\mathrm{p}<0.01$ ).

\section{DISCUSSION}

Although RA, a systemic autoimmune inflammatory disorder, primarily affects the synovial joints, it also damages other tissues and organs. RA affects women 3-5 times more often than men. ${ }^{(16)}$ Previous studies on RA in Taiwan reported a male to female ratio of approximately $1: 3 .{ }^{(17)}$

In the present study, which was based on the analysis of claims data, we found that of the total of 2,843 patients who 
Table I. Characteristics of patients with either rheumatoid arthritis (RA) or osteoarthritis (OA), who received unilateral arthroplasty, either total hip replacement or total knee replacement, among the 1,000,000 patients sampled from 1996 to 2010.

\begin{tabular}{|c|c|c|c|c|c|c|}
\hline \multirow[t]{2}{*}{ Characteristic } & \multicolumn{3}{|c|}{ Total hip replacement } & \multicolumn{3}{|c|}{ Total knee replacement } \\
\hline & RA patients & OA patients & p-value & RA patients & OA patients & p-value \\
\hline $1996-2000$ & $(n=10)$ & $(n=250)$ & & $(n=39)$ & $(n=1,109)$ & \\
\hline Gender & & & 0.75 & & & $0.04^{*}$ \\
\hline Male & $5(50.0)$ & $103(41.2)$ & & $4(10.3)$ & $269(24.3)$ & \\
\hline Female & $5(50.0)$ & $147(58.8)$ & & $35(89.7)$ & $840(75.7)$ & \\
\hline Age (yr) & $44.8 \pm 16.3$ & $58.6 \pm 13.1$ & $0.01^{*}$ & $54.4 \pm 12.4$ & $66.9 \pm 7.5$ & $<0.01^{*}$ \\
\hline $\mathrm{CCl}$ & & & $<0.01^{*}$ & & & $<0.01^{*}$ \\
\hline 0 & $0(0)$ & 234 (93.6) & & $0(0)$ & $978(88.2)$ & \\
\hline 1 & $10(100.0)$ & $13(5.2)$ & & $31(79.5)$ & $113(10.2)$ & \\
\hline$\geq 2$ & $0(0.0)$ & $3(1.2)$ & & $8(20.5)$ & $18(1.6)$ & \\
\hline Hospital grade & & & 0.29 & & & 0.22 \\
\hline Centre & $5(50.0)$ & $129(51.6)$ & & $25(64.1)$ & $554(50.0)$ & \\
\hline Regional & $4(40.0)$ & $54(21.6)$ & & $6(15.4)$ & $238(21.5)$ & \\
\hline ALOS (days) & $19.0 \pm 13.3$ & $9.7 \pm 3.6$ & 0.05 & $10.1 \pm 3.9$ & $10.7 \pm 4.6$ & 0.43 \\
\hline \multicolumn{7}{|l|}{ Cost (USD) } \\
\hline Laboratory examinations & $164 \pm 75$ & $88 \pm 41$ & $0.01^{*}$ & $86 \pm 39$ & $86 \pm 44$ & 0.97 \\
\hline Administered drugs & $218 \pm 103$ & $122 \pm 79$ & $0.02^{*}$ & $148 \pm 147$ & $125 \pm 92$ & 0.13 \\
\hline Surgery & $895 \pm 56$ & $850 \pm 92$ & 0.12 & $863 \pm 98$ & $869 \pm 164$ & 0.83 \\
\hline Total medical costs & $4,435 \pm 771$ & $3,542 \pm 508$ & $0.01^{*}$ & $3,851 \pm 400$ & $3,919 \pm 837$ & 0.61 \\
\hline \multicolumn{7}{|l|}{ Readmission (days) } \\
\hline$\leq 14$ & $0(0.0)$ & $7(2.8)$ & 0.59 & $0(0.0)$ & $40(3.6)$ & 0.23 \\
\hline $15-30$ & $0(0.0)$ & $8(3.2)$ & 0.57 & $1(2.6)$ & $24(2.2)$ & 0.87 \\
\hline $31-90$ & $0(0.0)$ & $9(3.6)$ & 0.54 & $2(5.1)$ & $48(4.3)$ & 0.81 \\
\hline 2001-2005 & $(n=23)$ & $(n=410)$ & & $(n=47)$ & $(n=2,026)$ & \\
\hline Gender $^{+}$ & & & 0.16 & & & 0.93 \\
\hline Male & $6(26.1)$ & $168(41.0)$ & & $12(25.5)$ & $528(26.1)$ & \\
\hline Female & $17(73.9)$ & $242(59.0)$ & & $35(74.5)$ & $1,497(73.9)$ & \\
\hline $\mathrm{CCl}$ & & & $<0.01^{*}$ & & & $<0.01^{*}$ \\
\hline 0 & $0(0.0)$ & $361(88.0)$ & & $0(0.0)$ & $1,723(85.0)$ & \\
\hline 1 & $20(87.0)$ & $43(10.5)$ & & $37(78.7)$ & $256(12.6)$ & \\
\hline$\geq 2$ & $3(13.0)$ & $6(1.5)$ & & $10(21.3)$ & $47(2.3)$ & \\
\hline Hospital grade & & & 0.84 & & & 0.41 \\
\hline Centre & $12(52.2)$ & $225(54.9)$ & & $21(44.7)$ & 787 (38.8) & \\
\hline Regional & $5(21.7)$ & $99(24.1)$ & & $15(31.9)$ & $577(28.5)$ & \\
\hline District & $6(26.1)$ & $86(21.0)$ & & $11(23.4)$ & $662(32.7)$ & \\
\hline ALOS (days) & $8.9 \pm 4.6$ & $8.4 \pm 3.6$ & 0.54 & $8.2 \pm 2.7$ & $8.4 \pm 3.2$ & 0.63 \\
\hline \multicolumn{7}{|l|}{ Cost (USD) } \\
\hline Laboratory examinations & $145 \pm 189$ & $91 \pm 52$ & 0.19 & $87 \pm 37$ & $84 \pm 42$ & 0.54 \\
\hline Administered drugs & $142 \pm 235$ & $83 \pm 59$ & 0.25 & $82 \pm 51$ & $84 \pm 97$ & 0.91 \\
\hline Surgery & $934 \pm 35$ & $875 \pm 57$ & $<0.01^{*}$ & $859 \pm 63$ & $868 \pm 110$ & 0.59 \\
\hline Total medical costs & $3,906 \pm 1,074$ & $3,379 \pm 386$ & $0.03^{*}$ & $3,596 \pm 290$ & $3,615 \pm 469$ & 0.79 \\
\hline \multicolumn{7}{|l|}{ Readmission (days) } \\
\hline$\leq 14$ & $2(8.7)$ & $7(1.7)$ & $0.02^{*}$ & $0(0.0)$ & $46(2.3)$ & 0.30 \\
\hline $15-30$ & $0(0.0)$ & $10(2.4)$ & 0.45 & $1(2.1)$ & $35(1.7)$ & 0.83 \\
\hline $31-90$ & $2(8.7)$ & $16(3.9)$ & 0.26 & $7(14.9)$ & $95(4.7)$ & $<0.01^{*}$ \\
\hline 2006-2010 & $(n=16)$ & $(n=531)$ & & $(n=60)$ & $(n=3,439)$ & \\
\hline Gender & & & 0.11 & & & 0.38 \\
\hline Male & $3(18.8)$ & $203(38.2)$ & & $12(20.0)$ & 857 (24.9) & \\
\hline Female & $13(81.3)$ & $328(61.8)$ & & $48(80.0)$ & $2,582(75.1)$ & \\
\hline Age (yr) & $60.9 \pm 12.0$ & $63.2 \pm 13.2$ & 0.50 & $64.0 \pm 10.1$ & $69.9 \pm 7.9$ & $<0.01^{*}$ \\
\hline \multicolumn{7}{|l|}{$\mathrm{CCl}$} \\
\hline 0 & $0(0.0)$ & 431 (81.2) & $<0.01^{*}$ & $0(0.0)$ & $2,627(76.4)$ & $<0.01^{*}$ \\
\hline 1 & $10(62.5)$ & 91 (17.1) & & $47(78.3)$ & 721 (21.0) & \\
\hline$\geq 2$ & $6(37.5)$ & $9(1.7)$ & & $13(21.7)$ & $91(2.6)$ & \\
\hline
\end{tabular}


Table I. (Continued...)

\begin{tabular}{|c|c|c|c|c|c|c|}
\hline \multirow[t]{2}{*}{ Characteristic } & \multicolumn{3}{|c|}{ Total hip replacement } & \multicolumn{3}{|c|}{ Total knee replacement } \\
\hline & RA patients & OA patients & p-value & RA patients & OA patients & p-value \\
\hline Hospital grade & & & 0.45 & & & $0.04^{*}$ \\
\hline Regional & $5(31.3)$ & $161(30.3)$ & & $21(35.0)$ & $1,191(34.6)$ & \\
\hline District & $2(12.5)$ & $136(25.6)$ & & $9(15.0)$ & $986(28.7)$ & \\
\hline ALOS (days) & $7.3 \pm 1.8$ & $7.3 \pm 2.5$ & 0.89 & $7.4 \pm 2.8$ & $7.4 \pm 2.6$ & 0.87 \\
\hline \multicolumn{7}{|l|}{ Cost (USD) } \\
\hline Laboratory examinations & $124 \pm 58$ & $107 \pm 59$ & 0.24 & $102 \pm 41$ & $103 \pm 61$ & 0.92 \\
\hline Administered drugs & $75 \pm 104$ & $53 \pm 74$ & 0.26 & $54 \pm 65$ & $55 \pm 82$ & 0.90 \\
\hline Surgery & $899 \pm 14$ & $900 \pm 32$ & 0.90 & $862 \pm 62$ & $909 \pm 88$ & $<0.01^{*}$ \\
\hline Total medical costs & $3,510 \pm 297$ & $3,401 \pm 366$ & 0.24 & $3,626 \pm 302$ & $3,772 \pm 455$ & $0.01^{*}$ \\
\hline \multicolumn{7}{|l|}{ Readmission (days) } \\
\hline$\leq 14$ & $0(0.0)$ & $8(1.5)$ & 0.62 & $2(3.3)$ & $59(1.7)$ & 0.34 \\
\hline $31-90$ & $0(0.0)$ & $17(3.2)$ & 0.47 & $4(6.7)$ & 115 (3.3) & 0.16 \\
\hline
\end{tabular}

Note: Categorical data is presented as no. (\%), while continuous data is presented as mean \pm standard deviation. Hospital charges were converted from Taiwan dollars to United States dollars using an exchange rate of 31.64:1, based on the average from 1996-2010. * $p$-value is statistically significant. ${ }^{D}$ ata on gender was not available for one patient. ALOS: average length of stay; $\mathrm{CCl}$ : Charlson comorbidity index

Table II. Analysis of the outpatient medical costs before and after surgery (3 months before surgery, and 3, 4-6 and 7-12 months after surgery) based on repeated measure ANOVA.

\begin{tabular}{|c|c|c|c|c|c|c|c|c|c|c|}
\hline \multirow[t]{2}{*}{ Parameter } & \multicolumn{5}{|c|}{ Total hip replacement } & \multicolumn{5}{|c|}{ Total knee replacement } \\
\hline & RA patients & p-value* & OA patients & p-value* & p-value ${ }^{+}$ & RA patients & p-value* & OA patients & p-value* & p-value ${ }^{+}$ \\
\hline 1996-2000 & $(n=10)$ & & $(n=250)$ & & & $(n=39)$ & & $(n=1,109)$ & & \\
\hline 3 mths after & $58 \pm 53$ & 1.00 & $77 \pm 96$ & 0.20 & 0.54 & $70 \pm 131$ & 0.40 & $82 \pm 100$ & $<0.01^{\S}$ & 0.48 \\
\hline 4-6 mths after & $43 \pm 55$ & 1.00 & $38 \pm 62$ & $<0.01^{\S}$ & 0.82 & $33 \pm 66$ & 1.00 & $38 \pm 71$ & $<0.01^{\S}$ & 0.66 \\
\hline $7-12$ mths after & $42 \pm 82$ & 1.00 & $51 \pm 98$ & 0.39 & 0.79 & $34 \pm 76$ & 1.00 & $46 \pm 104$ & $<0.01^{\S}$ & 0.47 \\
\hline p-value ${ }^{*}$ & 0.80 & & $<0.01^{\S}$ & & & 0.08 & & $<0.01^{\S}$ & & \\
\hline 2001-2005 & $(n=23)$ & & $(n=410)$ & & & $(n=40)$ & & $(n=2,026)$ & & \\
\hline 3 mths before & $90 \pm 178$ & - & $74 \pm 88$ & - & 0.41 & $59 \pm 66$ & - & $64 \pm 83$ & - & 0.72 \\
\hline 3 mths after & $91 \pm 94$ & 1.00 & $77 \pm 91$ & 1.00 & 0.49 & $73 \pm 119$ & 1.00 & $88 \pm 102$ & $<0.01^{\S}$ & 0.31 \\
\hline $4-6$ mths after & $30 \pm 62$ & 0.93 & $43 \pm 90$ & $<0.01^{\S}$ & 0.47 & $33 \pm 70$ & 1.00 & $36 \pm 75$ & $<0.01^{\S}$ & 0.74 \\
\hline $7-12$ mths after & $109 \pm 245$ & 1.00 & $58 \pm 138$ & 0.08 & 0.33 & $35 \pm 81$ & 1.00 & $51 \pm 117$ & $<0.01^{\S}$ & 0.34 \\
\hline p-value ${ }^{*}$ & 0.31 & & $<0.01^{\S}$ & & & 0.11 & & $<0.01^{\S}$ & & \\
\hline 3 mths before & $47 \pm 80$ & - & $68 \pm 80$ & - & 0.30 & $60 \pm 90$ & - & $71 \pm 86$ & - & 0.32 \\
\hline 3 mths after & $46 \pm 46$ & 1.00 & $74 \pm 86$ & 0.96 & 0.20 & $84 \pm 124$ & 1.00 & $94 \pm 99$ & $<0.01^{\S}$ & 0.43 \\
\hline 4-6 mths after & $24 \pm 38$ & 1.00 & $39 \pm 108$ & $<0.01^{\S}$ & 0.59 & $48 \pm 148$ & 1.00 & $35 \pm 79$ & $<0.01^{\S}$ & 0.53 \\
\hline $7-12$ mths after & $20 \pm 34$ & 0.81 & $43 \pm 104$ & $<0.01^{\S}$ & 0.38 & $77 \pm 342$ & 1.00 & $48 \pm 131$ & $<0.01^{\S}$ & 0.52 \\
\hline p-value ${ }^{*}$ & 0.20 & & $<0.01^{\S}$ & & & 0.53 & & $<0.01^{\S}$ & & \\
\hline
\end{tabular}

Note: All values, except for $\mathrm{p}$ values, are presented as mean \pm standard deviation. Hospital charges were converted from Taiwan dollars to United States dollars using an exchange rate of 31.64:1, based on the average exchange rate during 1996-2010. * $p$-value obtained using pairwise comparisons (after operation time period compared with 3 months before operation). ${ }^{\dagger} p$-value obtained using tests of between-subjects effects. ${ }^{\ddagger} p$-value obtained using tests of within-subjects effects. ${ }^{\circledR}$ Data is statistically significant

underwent THR, $49(1.72 \%)$ were RA patients; and of the total of 7,352 patients who underwent TKR, $146(1.99 \%)$ were RA patients. Comparing between the RA and OA patients in our study cohort, we found no significant differences in gender ratio in both the THR and TKR groups. In the present study, $71.4 \%$ of the RA patients who underwent THR were female, while $80.8 \%$ of the RA patients who underwent TKR were female. There was no significant difference among the different groups. The greater incidence of surgery performed in female RA patients parallels the overall greater incidence of RA in females in Taiwan.

Although RA is most commonly diagnosed in patients aged 40-50 years, onset can occur at any age. In the present study, the diagnosis of RA was confirmed based on the catastrophic illness claim data in the NHIRD. Application for a catastrophic illness certificate for RA requires thorough clinical and laboratory analysis and is based on the diagnostic criteria of the American Rheumatism Association. ${ }^{(18)}$ Our analysis indicated a significant difference in the age at registration for catastrophic illness between RA patients who underwent THR and RA patients who underwent TKR (51.4 years vs. 60.6 years; $p<0.01$ ) (data not shown). In addition, over the study's 15-year duration, the age at which RA patients received either THR (44.8 years) or TKR (54.4 years) was significantly lower than the age at which OA patients received either THR (58.6 years) or TKR (66.9 years).

Other than affecting the synovial joints, RA can also affect many other organs. It contributes to lung fibrosis, renal 
amyloidosis and atherosclerosis, and increases the risk of myocardial infarction and stroke. $\mathrm{CCl}$ is a well-established metric of the severity of comorbidities, ${ }^{(14,15)}$ and is used to measure the severity of RA and OA. The results of the present study indicate that RA patients who underwent either THR or TKR had significantly higher $\mathrm{CCl}$ scores than OA patients who underwent the same procedures. Additionally, our analysis of the total medical costs incurred indicated greater costs for THR in RA patients in 1996-2000 and 2001-2005, and lower costs for TKR in RA patients in 2006-2010. The hospital grades of the patients who underwent THR were found to be similar in both RA and OA patients $(p=0.64)$. However, with regard to the number of patients who had undergone TKR in 2006-2010 at large medical centres, the proportion of RA patients was significantly greater than the proportion of OA patients $(p<0.01)$.

In November 1997, BNHI implemented case payment for both THR and TKR procedures, contributing to the lower prices reported in our study. ${ }^{(19)}$ Previous studies reported a dramatic decline in the various measures of medical utilisation, particularly ALOS and total hospitalisation costs, after the implementation of a case payment system. ${ }^{(20,21)}$ In all three time periods, we found no significant difference in the ALOS of RA and OA patients who received either THR or TKR. In 1996-2000, the ALOS of RA patients who underwent THR was 9 days longer than that of OA patients who underwent THR, but this difference was not significant. The total medical cost during hospitalisation was independent of patient age, gender, $\mathrm{CCl}, \mathrm{ALOS}$ and hospital grade.

The costs of laboratory examinations and administered drugs were only higher for RA patients who underwent THR during 1996-2000 ( $p=0.01$ and $p=0.02$, respectively). This is probably because RA patients who have to undergo surgery usually have severely deformed joints and often are on analgesics and disease-modifying antirheumatic drugs (DMARDs), which can be expensive. In 2001-2005, the cost of surgery for THR was significantly higher for RA patients than for OA patients, while in 2006-2010, the cost of surgery for TKR was significantly higher for RA patients than for OA patients. This significantly higher cost of surgery in RA patients scheduled to undergo either THR or TKR is probably due to: (a) RA patients requiring soft tissue release for joint flexion contracture, and devitalised inflammatory synovial tissue is costly; and (b) the more complex surgery in RA patients. The results of the present study also showed significant within-subject effects for OA patients who underwent THR and TKR were present in all three time periods (all $p<0.01$ ).

A previous study by March et $\mathrm{al}^{(22)}$ reported that the cost and service usage of THR and TKR decreased over the first postoperative year in RA patients. RA patients generally receive more pre- and postoperative outpatient care than OA patients due to the serious nature of RA. Treatment of RA patients using painkillers and DMARDs, as well as rehabilitation for flexion contracture of joints, may also lead to greater risk of poor wound healing, infection and dislocation, thereby increasing costs. ${ }^{(6,23,24)}$ Another study on RA in Australian patients by March et al reported that RA patients incurred significantly greater costs for THR and
TKR than OA patients. ${ }^{(9)}$ Schrama et al reported that the risk of revision for infection after TKR was higher in RA patients than in OA patients. ${ }^{(25)}$ In contrast, a study by da Cunha et al concluded that, relative to patients with primary OA or OA secondary to noninflammatory disease, patients with RA did not have increased risk for perioperative infection in THR and TKR, which is possibly due to the low rates of infection in both study groups. ${ }^{(26)}$ Previous studies indicate that RA patients with lower extremity arthroplasty have longer rehabilitation stays and less improvement in lower functional independence measures. ${ }^{(27,28)}$ Nevertheless, the flexion contracture of RA patients is expected to resolve over time with physiotherapy and continual loosening of the soft tissues.

The present study was not without limitations. As it utilised statistical data from NHIRD, analysis of implant brands, complications related to surgery, operation time, amount of blood lost during surgery, clinical characteristics of the disease and private payment of medical charges not covered by National Health Insurance was not possible as this information was not available in the database. Another limitation related to the use of this database is that it did not allow detailed investigations of individual patients. As the time of surgery and severity of the disease are related to the National Health Insurance System in Taiwan, there may have been some differences in preoperative costs. Finally, the present study provided an analysis of direct costs, but not indirect costs. It is generally agreed that over an RA patient's lifetime, the direct medical costs of an RA patient are about two times of the indirect costs. ${ }^{(29)}$

A major strength of the present study is that it was a large systemic comparison of THR and TKR in RA and OA patients over a period of 15 years. Moreover, all diagnoses were performed and confirmed according to ICD-9-CM guidelines, the sample sizes were sufficiently large to allow meaningful comparisons, and the results reflect actual medical utilisation of THR and TKR surgeries for RA and OA patients under the governance of the National Health Insurance of Taiwan. The results of the present study provide a basis for comparisons of the use of THR and TKR in RA and OA patients from different populations.

In conclusion, our comparison of RA and OA patients in Taiwan who underwent either THR or TKR indicated that RA patients were younger and used significantly more medical resources before, during and after hospitalisation.

\section{DICLAIMER}

Although the present study is based in part on data from NHIRD (provided by the Bureau of National Health Insurance, Department of Health, Taiwan), which is managed by the National Health Research Institutes, the interpretations and conclusions contained herein do not represent those of the Bureau of National Health Insurance, Department of Health, or the National Health Research Institutes.

\section{REFERENCES}

1. Chang RW, Pellisier JM, Hazen GB. A cost-effectiveness analysis of total hip arthroplasty for osteoarthritis of the hip. JAMA 1996; 275:858-65.

2. Lajas C, Abasolo L, Bellajdel B, et al. Costs and predictors of costs in rheumatoid arthritis: a prevalence-based study. Arthritis Rheum 2003; 49:64-70. 
3. Gibofsky A. Epidemiology, pathophysiology, and diagnosis of rheumatoid arthritis: A Synopsis. Am J Manag Care 2014; 20(7 Suppl):S128-45.

4. Silman AJ, MacGregor AJ, Thomson W, et al. Twin concordance rates for rheumatoid arthritis: results from a nationwide study. Br J Rheumatol 1993; 32:903-7.

5. Bellamy N, Duffy D, Martin N, Mathews J. Rheumatoid arthritis in twins: A study of aetiopathogenesis based on the Australian Twin Registry. Ann Rheum Dis 1992; 51:588-93.

6. Lu SR, Lin HY, Lin KC, Lin HR. Quality of life in middle-aged and older Taiwanese patients with rheumatoid arthritis. J Nurs Res 2008; 16:121-30

7. Lawrence RC, Helmick CG, Arnett FC, et al. Estimates of the prevalence of arthritis and selected musculoskeletal disorders in the United States. Arthritis Rheum 1998; 41:778-99.

8. Centers for Disease Control and Prevention. National Arthritis Action Plan: A Public Health Strategy. Atlanta, GA: CDC, 1999.

9. March LM, Barcenilla AL, Cross MJ, et al. Costs and outcomes of total hip and knee joint replacement for rheumatoid arthritis. Clin Rheumatol 2008; 27:1235-42.

10. Canadian Institute for Health Information. Waiting for Health Care in Canada: What We Know and What We Don't Know. Available at: https://secure.cihi.ca/free_products/WaitTimesReport_06_e.pdf. Accessed November 3, 2014.

11. National Joint Registry for England and Wales. 3rd Annual Clinical Report Available at: http://www.njrcentre.org.uk/NjrCentre/Portals/0/Documents/ England/Reports/NJR_CR_3.pdf. Accessed November 3, 2014.

12. Wen B, Li H, Lu D, et al. Genetic evidence supports demic diffusion of Han culture. Nature2004; 431:302-5.

13. Lovaasen KR, Schwerdtfeger J. ICD-9-CM Coding: Theory and Practice. Philadelphia: WB Saunders, 2007.

14. Chu YT, Wu SC. [A review of claims-based comorbidity measures]. Taiwan J Public Health 2010; 29:8-21. Chinese.

15. Wu SC, Chien LN, Ng YY. [Using the comorbidity index to evaluate the medical utilization and outcomes of total hip replacement through analyzing National Insurance claimed data]. Taiwan J Public Health 2004; 23:121-9. Chinese.

16. Majithia V, Geraci SA. Rheumatoid arthritis: diagnosis and management. Am J Med 2007; 120:936-9.

17. Chao YC, Huang $\mathrm{KY}$, Tung $\mathrm{CH}$, et al. [Treatment of DMARD-resistant active rheumatoid arthritis patients with leflunomide: Central Taiwan experience]. Formosan J Rheumatol 2007; 21:57-63. Chinese.

18. Arnett FC, Edworthy SM, Bloch DA, et al. The American Rheumatism Association 1987 revised criteria for the classification of rheumatoid arthritis. Arthritis Rheum 1988; 31:315-24.

19. Chang CC, Hung WF. [Impact of NHI Payment on Medical Utilization of Total Hip Replacement and Total Knee Replacement]. Taiwan J Public Health 2001; 20:440-50. Chinese.

20. Healy WL, lorio R. Implant selection and cost for total joint arthroplasty: conflict between surgeons and hospitals. Clin Orthop Relat Res 2007; 457:57-63.

21. Mitsuyasu S, Hagihara A, Horiguchi H, Nobutomo K. Relationship between total arthroplasty case volume and patient outcome in an acute care payment system in Japan. J Arthroplasty 2006; 21:656-63.

22. March L, Cross M, Tribe K, et al. Cost of joint replacement surgery for osteoarthritis: the patients' perspective. J Rheumatol 2002; 29:1006-14.

23. Ravi B, Escott B, Shah PS, et al. A systematic review and meta-analysis comparing complications following total joint arthroplasty for rheumatoid arthritis versus for osteoarthritis. Arthritis Rheum 2012; 64:3839-49.

24. Ravi B, Croxford R, Hollands S, et al. Increased risk of complications following total joint arthroplasty in patients with rheumatoid arthritis. Arthritis Rheumatol 2014; 66:254-63.

25. Schrama JC, Espehaug B, Hallan G, et al. Risk of revision for infection in primary total hip and knee arthroplasty in patients with rheumatoid arthritis compared with osteoarthritis: a prospective, population-based study on 108,786 hip and knee joint arthroplasties from the Norwegian Arthroplasty Register. Arthritis Care Res (Hoboken) 2010; 62:473-9.

26. da Cunha BM, de Oliveira SB, Santos-Neto L. Incidence of infectious complications in hip and knee arthroplasties in rheumatoid arthritis and osteoarthritis patients. Rev Bras Reumatol 2011; 51:609-15.

27. Nguyen-Oghalai TU, Ottenbacher KJ, Caban M, et al. The impact of rheumatoid arthritis on rehabilitation outcomes after lower extremity arthroplasty. J ClinRheumatol 2007; 13:247-50.

28. Chung HK, Choi YJ, Choi CH, Kim JH, Roh KW. [Radiologic evaluation of improved residual flexion contracture after TKRA in rheumatoid arthritis]. J Korean Knee Soc 1999; 11:26-31. Korean.

29. Maetzel A, Li LC, Pencharz J, et al. The economic burden associated with osteoarthritis, rheumatoid arthritis, and hypertension: a comparative study. Ann Rheum Dis 2004; 63:395-401. 